\title{
Reconsidered estimates of the 10th order QED contributions to the muon anomaly
}

\author{
A.L. Kataev \\ Institute for Nuclear Research of the Academy of Sciences of Russia, \\ 117312, Moscow, Russia
}

\begin{abstract}
The problem of estimating the 10th order QED corrections to the muon anomalous magnetic moment is reconsidered. The incorporation of the recently improved contributions to the $\alpha^{4}$ and $\alpha^{5}$-corrections to $a_{\mu}$ within the renormalization-group inspired scheme-invariant approach leads to the estimate $a_{\mu}^{(10)} \approx 643(\alpha / p i)^{5}$. It is in good agreement with the estimate $a_{\mu}^{(10)}=$ $663(20)(\alpha / \pi)^{5}$, obtained by Kinoshita and Nio from the numerical calculations of 2958 10-th order diagrams, which are considered to be more important than the still uncalculated 6122 10th-order $m_{\mu} / m_{e}$-dependent vertex graphs, and 126725 -loop diagrams, responsible for the mass-independent constant contribution both to $a_{\mu}$ and $a_{e}$. This confirms Kinoshita and Nio guess about dominance of the 10-th order diagrams calculated by them. Comparisons with other estimates of the $\alpha^{5}$ - contributions to $a_{\mu}$, which exist in the literature, are presented.
\end{abstract}

PACS: 12.20.Ds,; 12.38.Bx; 13.40.Em; 14.60.Ef 


\section{Introduction}

The problem of estimating higher order perturbative corrections to the anomalous magnetic moments was first formulated by Feynman. In his 1961 talk at one of the Conferences from the Solvey series he mentioned "As a special challenge, is there any method of computing the anomalous magnetic moment of the electron which, on the first rough approximation, gives a fair approximation to the $\alpha$-term and a crude one to $\alpha^{2}$ term, yielding a rough estimate to $\alpha^{3}$ and beyond ?" [1]. This challenge was proposed when only two corrections from the perturbative series for the anomalous magnetic moment of the electron $a_{e}$

$$
a_{e}=\sum_{i \geq 1} A_{i}\left(\frac{\alpha}{\pi}\right)^{i}
$$

were known. The coefficient

$$
A_{1}=\frac{1}{2}
$$

was calculated by Schwinger [2, while the numerical value of the second coefficient

$$
A_{2}=-0.328478965 \ldots
$$

is known from the independent analytical calculations of Sommerfield [3], Petermann 4], and Terentiev [5]. An attempt to answer the Feynman challenge was made in Ref. [6]. Using dispersion theory the authors of Ref. [6] managed to reproduce $90 \%$ of the $A_{2}$-term and presented the following estimate of the third coefficient of the expansion of $a_{e}$ in powers of $(\alpha / \pi)$ :

$$
A_{3} \approx+0.15 \ldots
$$

An analog of the approach of Ref. [6] was developed in Ref. 7]. By fixating of a cut-off in one of the basic relations in the dispersion theory approach, they were able to reproduce the fourth order term $A_{2}(\alpha / \pi)^{2}$ approximately [7] and this led to the estimate for the $(\alpha / \pi)^{3}$ coefficient

$$
A_{3} \approx+0.13
$$

in agreement with the estimate of Eq. (41). Thus, initially in extracting information on the value of $(\alpha / \pi)^{3}$-contributions to $a_{e}$ the dispersion technique was considered the proper tool for estimating higher-order corrections [7]. However, it was realized later on, that this method cannot give information on the total value of the 6 -th order contributions to $a_{e}$, since the contribution of several sets of three-loop diagrams cannot be modeled within this approach. The first contribution, missed in the estimating procedures used in Refs. [6, 7], is the diagram with external electron vertex and internal photon line, dressed by two electron loops. It was analytically calculated in Ref. [8]. Another important contribution, not taken into account in the estimates of Refs. [6, 7], is the subset of three-loop diagrams with a light-by-light-scattering subgraph. This contribution was first evaluated numerically in Ref. [9], and analytically almost twenty years later in Ref. [10. The analytical calculations of all 6-th order diagrams were completed by Laporta and Remiddi in Ref. [11] with the help of the computer symbolic manipulations technique. The numerical form of the results obtained in Ref. [1] read :

$$
A_{3}=+1.181241456 \ldots
$$


Thus, the estimates of the 6-th order contribution to $a_{e}$, made in Refs. 6, 7, gave the correct sign and pushed ahead the interest in calculating the graphs not included in these estimates. In its turn, the desire to perform complicated calculations led to the development of the symbolic manipulation computer programs (for a review see Ref. [12]), which played an essential role in obtaining final expression for the 6-th order corrections to $a_{e}[11$ in the analytical form. Note, that the analytical calculation of the order $\alpha^{3}$ correction to $a_{e}$, completed by Remiddi and Laporta in Ref. [11, was continuing over 27 years after the first analytical calculation of the set 3-loop of diagrams performed in Ref. [8]. Several theoreticians, namely Barbieri, Caffo, Levine, Remiddi, Roskies and Laporta, contributed to the calculations of different diagrams during this long-termed project (see citations of the related works in Ref. [11]). Thus, the problem discussed at the end of 1960-s in the Theory Division of INP, Gatchina of starting from scratch and completing calculations of this correction rather fast [13, was really unsolvable problem. This was correctly understood by the members of this theoretical group after counting the number and drawing the typical representatives of the 3-loop diagrams, which are contributing to $a_{e}$.

\section{Previous status of the estimates of the 10-th order QED corrections to $a_{\mu}$}

The story of the estimation of the value of the 10-th order contributions to the muon anomalous magnetic moment $a_{\mu}$, developed in a rather similar way to the studies of the QED corrections to $a_{e}$ described above. The first estimate, namely

$$
a_{\mu}^{(10)} \approx 570(140)\left(\frac{\alpha}{\pi}\right)^{5}
$$

was given in Ref. [14. It was based on the explicit numerical calculations of the diagrams from the set of the 10-th order contributions to $a_{\mu}$ with the electron light-by-light-type subgraph, which has internal photon lines, dressed by two electron bubbles (an example of these diagrams is shown in Fig.1).

The idea to evaluate the contributions of these diagrams first of all was based on the observation that the most important contributions to the 6-th order and 8-th order QED corrections to $a_{\mu}$ came from light-by-light type graphs with an internal electron loop insertion, coupled to the external muon line by three photon propagators. At 6-th order the direct numerical calculations of these three-loop diagrams with electron light-by-light scattering insertions were calculated first in Ref. 9] and essentially improved later on in Ref. [15]. It is worth mentioning here that this important contribution is now known analytically [16. Moreover, this expression was confirmed and even improved by calculating analytically several additional terms, proportional to $\left(m_{e} / m_{\mu}\right)^{2 \mathrm{k}}(3 \leq \mathrm{k} \leq 5)$ in Ref. [17. At the four-loop level the dominating role of the "light-by-light-type" effects were revealed in the process of the numerical calculations of Ref. [14.

However, as in the case of the 6 -th order correction to $a_{e}$, the estimates of the 10-th order 


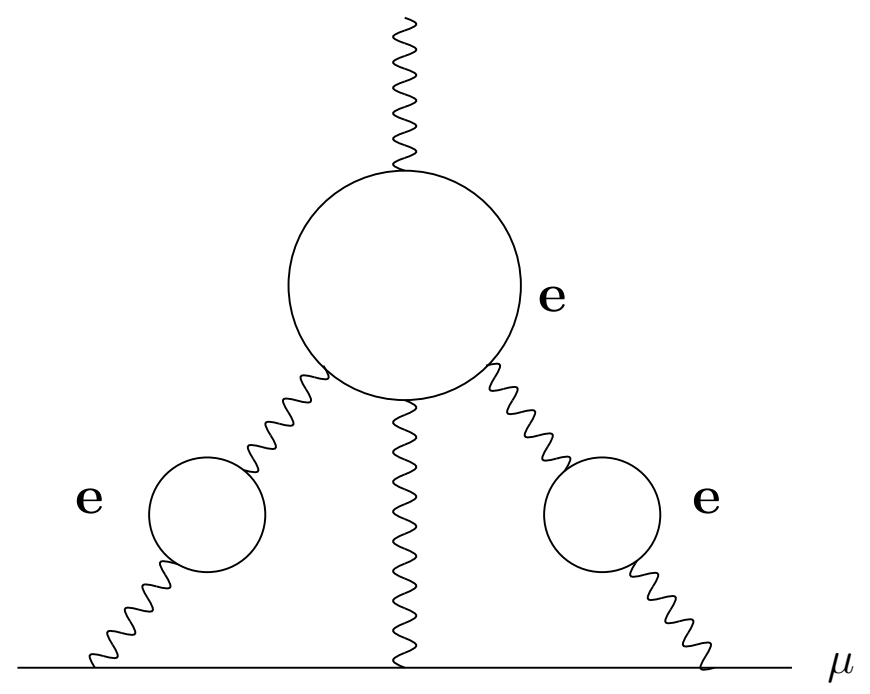

Figure 1: The example of diagram, which was explicitly calculated in the process of deriving first estimate of the 10-th order QED correction to $a_{\mu}$.

corrections to $a_{\mu}$ of Eq. (17) demonstrated that there are additional important contributions to $a_{\mu}$, which first appear at five-loop order. Indeed, as was discussed in Ref. 18] the subsets of diagrams depicted in Fig.2 and Fig.3 may also generate sizable additional contributions to the 10-th order QED corrections to $a_{\mu}$.

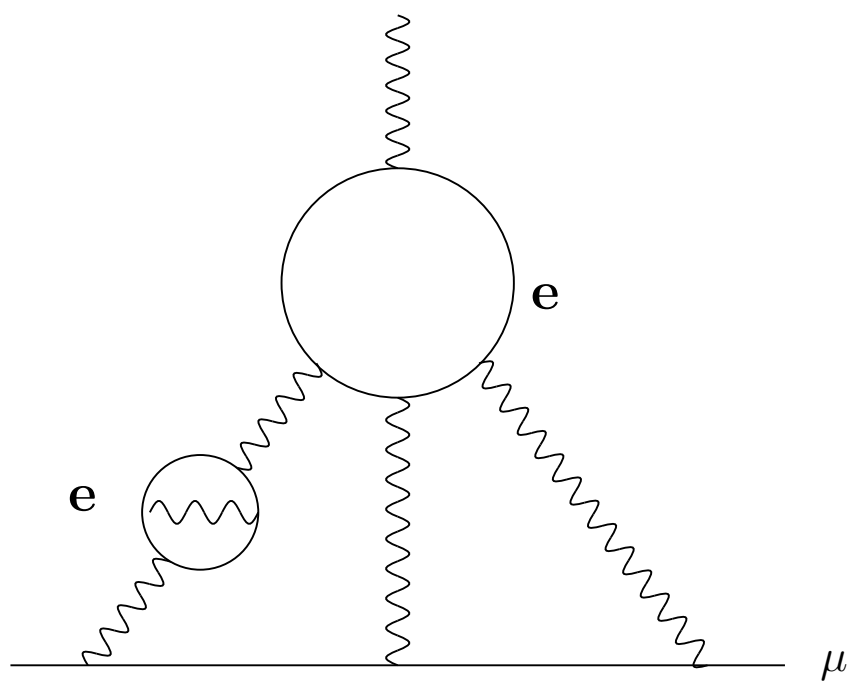

Figure 2: The diagram, estimated first in Ref.[18] using the RG-based arguments.

The diagrams of Fig.2 contain two-loop photon vacuum polarization insertions into one of the three internal photon lines, which connect electron light-by-light type subgraphs with the external muon line. To find the approximate value of this contribution the asymptotic expression for the two-loop photon vacuum polarization function was taken into account. As a 


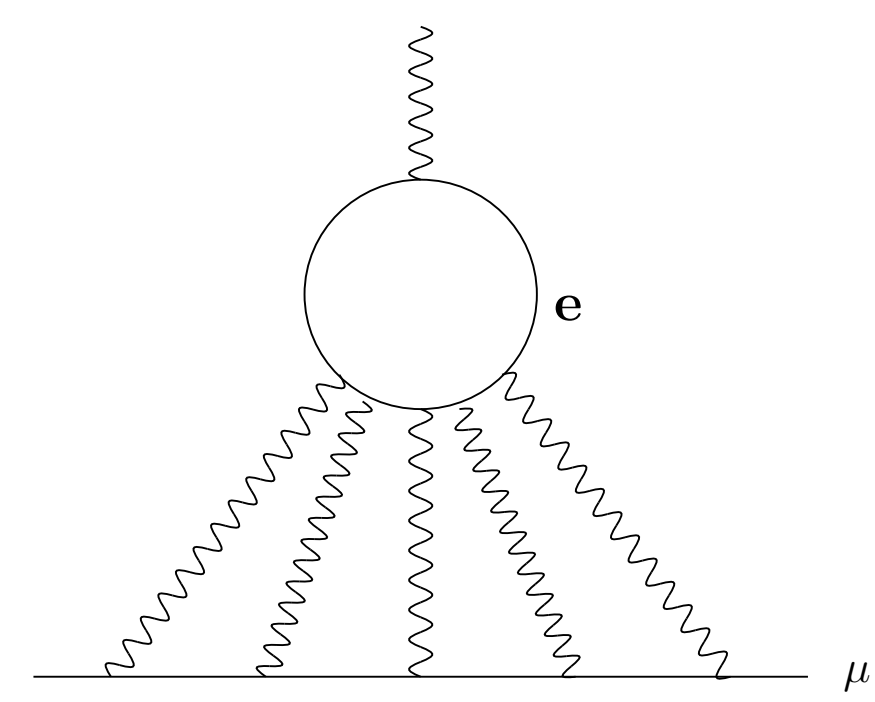

Figure 3: The example of typical diagram, which appear first at the 10-th order.

result, the following estimate was obtained [18]:

$$
a_{\mu}^{(10)}(\text { Fig. } 2) \approx 176(35)\left(\frac{\alpha}{\pi}\right)^{5} .
$$

Another important additional contribution to $a_{\mu}^{(10)}$ comes from a set of diagrams, which appear first at this 5-loop level. They contain an internal electron loop, connected by five internal photon lines to the external muon line (see Fig.3). It was shown in Ref. [19], that the expression for this diagram has a structure typical of the class of light-by-light-type-scattering diagrams, namely, it contains a $\ln \left(\mathrm{m}_{\mu} / \mathrm{m}_{\mathrm{e}}\right)$-term, which has a non-renormalization group origin. The general expression for this contribution can be written in the following form [19]:

$$
a_{\mu}^{(10)}(\text { Fig. } 3) \approx\left(\mathbf{C}_{\mathbf{1}} \pi^{4} \ln \left(\mathrm{m}_{\mu} / \mathrm{m}_{\mathrm{e}}\right)+\mathbf{C}_{\mathbf{2}}\right)\left(\frac{\alpha}{\pi}\right)^{5} .
$$

The numerical value of the coefficient $\mathbf{C}_{\mathbf{1}}=0.438$, cited in Ref. [18, comes from calculations of Ref. 20]. Assuming, that the unknown constant term $\mathbf{C}_{2}$ of Eq.(9) is not very large, the author of Ref. [18] estimated this contribution as:

$$
a_{\mu}^{(10)}(\text { Fig. } 3)=185(85)\left(\frac{\alpha}{\pi}\right)^{5},
$$

The value for one more set of the five loop light-by-light-type diagrams, not considered in Ref.[14, and depicted in Fig. 4, was estimated in Ref.[18] as

$$
a_{\mu}^{(10)}(\text { Fig. } 4)= \pm 40\left(\frac{\alpha}{\pi}\right)^{5},
$$

without giving any theoretical arguments. 


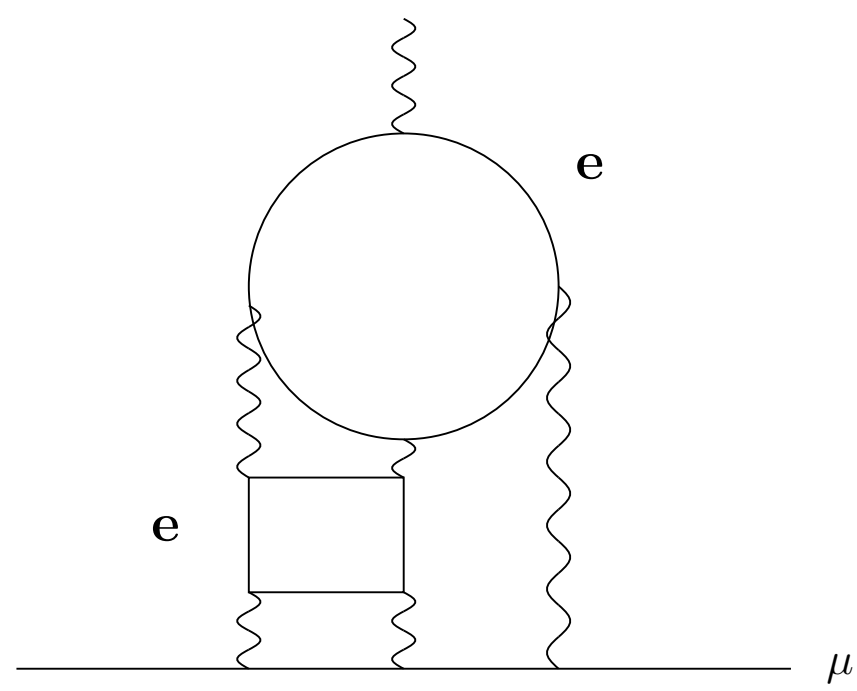

Figure 4: The diagram, which was first considered in Ref. [18] in the process of deriving the second estimate of the 10-th order correction to $a_{\mu}$.

Combining Eq.(17), Eq.(8), Eq.(10) and Eq.(11), the author of Ref. [18] presented the following modified estimate for the 10th-order QED contributions to $a_{\mu}$

$$
a_{\mu}^{(10)}(\operatorname{Ref} .[18])=930(170)\left(\frac{\alpha}{\pi}\right)^{5}
$$

which was used in a number of phenomenological works on the subject (see e.g. Ref.[21]).

Some time later the question of the contribution of the 10-th order QED corrections to $a_{\mu}$ was analyzed in Ref. 22] using the approaches closely related to the renormalization-group (RG) method, described in detail in Ref. [23, namely the principle of minimal sensitivity [24] and the effective charges (ECH) approach [25, 26] (for the analysis of theoretical problems and definite phenomenological applications of the ECH-approach see Refs. [27]- 29]).

In the process of applying the method of Ref. [22, tested in QCD at the $\alpha_{s}^{3}$-level in Ref. [30, explicit calculations of the four-loop contributions to $a_{\mu}$ [14] and of the QED RG $\beta$-function in the on-shell-scheme [31] were used. The expression of Eq.(10) for the new set of diagrams of Fig.3, first appear in 10-th order, was added to the numbers which result from the separate application of the renormalization-group inspired approach of Ref. [22] to the set of graphs with external muon vertex (Set I in the notations of Ref. [22], see Fig. 5), and to the set of diagrams which is generated by the three-loop light-by-light-scattering subgraphs with three internal photon propagators coupled to the external muon line ( Set II in the notations of Ref. [22]).

The estimate of the 10-th order contribution from the Set I of diagrams obtained in Ref. [22], is

$$
a_{\mu}^{(10)}(\text { Set I }) \approx 50\left(\frac{\alpha}{\pi}\right)^{5} .
$$




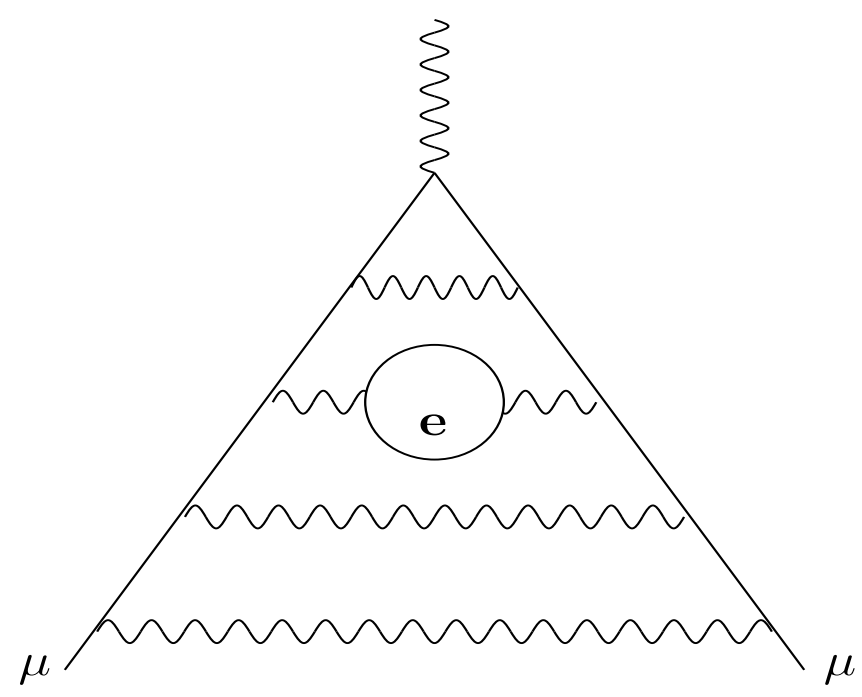

Figure 5: The example of the diagrams which produce RG-controllable $\ln \left(\mathrm{m}_{\mu} / \mathrm{m}_{\mathrm{e}}\right)$-terms in the expression for $a_{\mu}$.

The second part of the whole 10-th order estimate of Ref. 22, which came from the consideration of the light-by-light-type graphs, was

$$
a_{\mu}^{(10)}(\text { Set II }) \approx 520\left(\frac{\alpha}{\pi}\right)^{5}
$$

It should be compared with the estimate, based on summing the number in Eq.(7), which comes from the direct 10-th order calculations of Ref. [14, and the number of Eq.(8), which is the result of application of renormalization-group inspired formalism in the way, different from our work.One can see, that within existing huge error-bars this result of the developed in Ref. [22] RG-inspired approach (namely, Eq.(14)) is in satisfactory agreement with sum of Eqs.(7) and Eq.(8).

The total 10-th order estimate of Ref. [22, namely

$$
a_{\mu}^{(10)}(\mathrm{tot}) \approx 750\left(\frac{\alpha}{\pi}\right)^{5}
$$

is just the sum of Eq. (13), Eq.(14) and Eq. (10). It should be compared with the estimate, given in Eq. (12). We will see later on, that the improved explicit calculations of the 10-th order corrections of Ref. 32] are in better agreement with the estimate of Eq. (15), obtained in Ref. 22], than with estimate of Eq.(12), proposed in Ref. [32. Moreover, the improved RG-inspired analysis will allow us to get even the number, which is even closer to the results of the improved calculations of Ref.[32], than Eq.(15). 


\section{The improved renormalization-group inspired estimates}

\subsection{Preliminaries}

During several the last years new results of calculations of a number of 8-th and 10-th order QED contributions to $a_{\mu}$ have appeared in the literature (see the review of Ref. 33. and the original works of Refs. 34], 35] and [32]). In view of this, it is interesting to study the predictive possibilities of the scheme-invariant methods, incorporating new 4-loop and 5-loop exact results into the machinery of the approach of Ref. 22]. This will allow us to understand whether the relative predictive success of the RG-inspired method of Refs. 22, [30] is an accident or it has a more solid background.

The total value of the 5-loop 10-th order QED correction to $a_{\mu}$ involves 21752 diagrams. Among them are 12672 mass-independent graphs, contributing both to $a_{e}$ and $a_{\mu}$. They should not give huge coefficients to the $O\left(\alpha^{5}\right)$-term of the perturbative series for the electron and muon anomalies. Among the remaining 9080 5-loop diagrams, 2958 were considered to be more important than 6122 vertex graphs. [32]. They contain $\ln \left(\mathrm{m}_{\mu} / \mathrm{m}_{\mathrm{e}}\right)$ terms, which arise from light-by-light-scattering subgraphs and vacuum-polarization insertions. Numerical calculations, performed by Kinoshita and Nio of the dominant 2958 10-th order graphs, resulted in the following more definite estimate of the 10-th order QED contribution to $a_{\mu}$

$$
a_{\mu}^{(10)}(\operatorname{Ref} .[32])=663(20)\left(\frac{\alpha}{\pi}\right)^{5}
$$

As was already mentioned above, it turned out to be closer to the estimate of Ref.[22], than the one of Ref. 18] (compare Eq.(16) with Eq.(15) and Eq.(12)).

However, since the new explicit results of calculations of the 10-th order corrections to $a_{\mu}$ appeared in Ref. 32, it is the time to improve the knowledge about results of applications of the RG-inspired approach of Ref. [22]. At the first stage of these studies the preliminary numerical expression for the set of diagrams of Fig.3, announced in Ref. 37, 38] was taken into account [39]. The reduction of the overall value of the 10-th order estimates given in Ref. [22] and their striking agreement with the preliminary results of the direct numerical calculations, announced in Ref. [37, were observed [39].

It is quite understandable, that more careful application of the RG inspired scheme-invariant approach at the 10-th order necessitates the consideration of several additional effects. They are:

1. the estimates of the 10-th order light-by-light-type (l-b-l) contributions, which is generated by including the new values of the 8-th order QED corrections, improved in the process

of calculations of the Ref. [34, 35]. This improvement result in the following shift of the 8-th order light-by-light scattering terms:

$$
\Delta^{(8)}=a_{\mu}^{(8)}(\mathrm{l}-\mathrm{b}-\mathrm{l},[34])-a_{\mu}^{(8)}(\mathrm{l}-\mathrm{b}-\mathrm{l},[14]) \approx 5.2\left(\frac{\alpha}{\pi}\right)^{4} ;
$$


2. the RG motivated estimates for the subset of 10-th order graphs, which is generated by 8-th order graphs with internal light-by-light-type insertion, coupled to the external muon vertex by four internal photon lines (see Fig. 6). In view of the absence of explicit 10-th order results, this estimate was not considered separately in Ref. [22, but was included in the overall estimate of Eq.(14);

3. the explicit results for the 10-th order corrections from the diagrams of Fig. 3 and Fig. 4 published in Ref. 32.

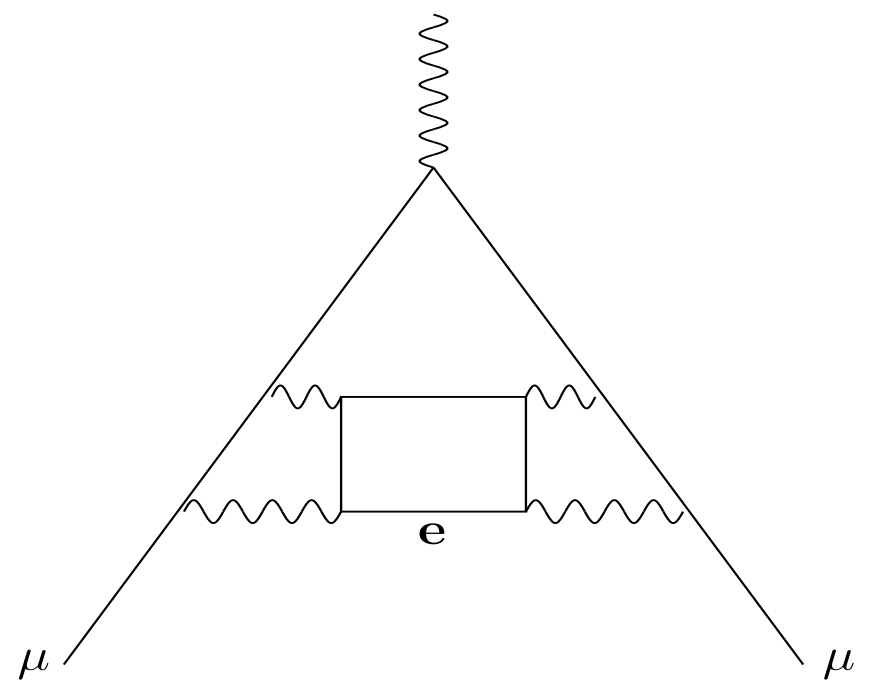

Figure 6: The 8-th order diagram, which is generating one of the gauge-invariant sets of diagrams, contributing to $a_{\mu}$.

\subsection{Technical considerations}

In this Section we will analyze all the effects discussed above.

Let us first recall the expressions for the coefficients of the series we will be dealing with.

The QED contributions to $a_{\mu}=\left(g_{\mu}-2\right) / 2$ can be defined by summing two perturbative series. The first one is the QED expression for the electron anomalous magnetic moment $a_{e}=\left(g_{e}-2\right) / 2$, which was discussed in the Introduction. Rigorously speaking, it has the following form

$$
\begin{aligned}
a_{e} & =\sum_{i \geq 1}^{4} A_{i} a^{i}+O\left(a^{2} m_{e} / m_{\mu}\right)+O\left(a^{2} m_{e} / m_{\tau}\right) \\
& =0.5 a-0.328478965 \ldots a^{2}+1.181241456 \ldots a^{3}-1.7283(35) a^{4} \\
& +O\left(a^{2} m_{e} / m_{\mu}\right)+O\left(a^{2} m_{e} / m_{\tau}\right)
\end{aligned}
$$


where $a=\alpha / \pi$, and the most precise numerical value for the coefficient $A_{4}$ was obtained in Ref. 35. It should be stressed that the available theoretical expression for $a_{e}$ agrees with the most recent experimental value of $a_{e}$ [36] at the level of $10^{-10}$ (for the most recent discussions see Ref. 32]). At this level of precision the mass dependent corrections, which all are $\leq 3 \times 10^{-12}$ [32, 40], can be safely neglected.

Other, more sizable perturbative QED contributions, to $a_{\mu}$ can be combined in the series, which is defined as

$$
a_{\mu}-a_{e}=A_{2}\left(m_{\mu} / m_{e}\right)+O\left(m_{e} / m_{\mu}\right)+O\left(m_{\mu} / m_{\tau}\right) .
$$

The numerical coefficients for $A_{2}\left(m_{\mu} / m_{e}\right)$ read

$$
A_{2}\left(m_{\mu} / m_{e}\right)=1.0942583111(84) a^{2}+22.86838002(20) a^{3}+132.6823(72) a^{4}+O\left(a^{5}\right)
$$

The $O\left(m_{e} / m_{\mu}\right)$ and $O\left(m_{\mu} / m_{\tau}\right)$-corrections to Eq.(19]) are rather small (see e.g. Refs. 33], 34]). Since we are interested in estimating order $O\left(a^{5}\right)$-effects in the series with increasing positive coefficients (see Eq.(20)), we will neglect these small corrections in all further discussions.

It should be stressed, that all diagrams contributing to $a_{\mu}$ at various orders of perturbation theory form gauge-invariant independent sets with different topological structure. In view of this and in accordance with the proposal of Ref. 41] to consider the contributions to coefficients of the perturbative series to gauge-invariant sets separately we will divide the expression for the 10th-order QED contribution to $a_{\mu}$, defined by five-loop diagrams, into five independent gauge-invariant contributions, namely

$$
\Delta_{(10)}=\Delta_{(10)}^{(I)}+\Delta_{(10)}^{(I I)}+\Delta_{(10)}^{(I I I)}+\Delta_{(10)}^{(I V)}+\Delta_{(10)}^{(V)}
$$

where Set I includes the diagram with external muon vertex and no light-by-light type graphs (see Fig.5), and Set II includes the diagrams with light-by-light type subgraph, which has one external and three internal photon propagators (this set contains the diagrams of the subsets of Fig.1 and Fig.2). The generating graph of Set III contains a "box"-type light-bylight-scattering insertion into muon vertex, coupled to the external muon legs by four internal photon propagators (see Fig. 6). Set IV is generated by light-by-light scattering subgraphs with one external and five internal muon lines (see Fig.3). Set V is generated by a "box"-type internal insertion into the three-loop light-by-light-scattering subgraph. This "box" subgraph is coupled to the internal photon lines (see Fig.6).

It is known that in the case of the pertrurbative series for $a_{\mu}$ the analytical expressions for all high-order diagrams with internal electron loop insertions contain $\ln \left(\mathrm{m}_{\mu} / \mathrm{m}_{\mathrm{e}}\right)$-terms. Parts of them, namely the ones which are coming from vacuum polarization electron bubble insertions into internal photon lines, are governed by the RG method. However, the expressions for the leading diagrams, which are forming sets with subgraphs, generated by the effects of light-by-light scattering on electron loops, contain $\ln \left(\mathrm{m}_{\mu} / \mathrm{m}_{\mathrm{e}}\right)$-terms which are not governed by the RG (typical examples are the diagrams of Fig.3 and other diagrams which are generating Set II, Set III, Set IV and Set V). The analytical expressions for the coefficients of these non-RG controllable $\ln \left(\mathrm{m}_{\mu} / \mathrm{m}_{\mathrm{e}}\right)$-terms in the diagrams from Set II, Set IV and Set V are proportional to 
sizable $\pi^{2}$-factor (see e.g. Refs. [42, [19] and Ref. [43] for the detailed discussions). Unfortunately, nothing is known analytically about the coefficients of the $\ln \left(\mathrm{m}_{\mu} / \mathrm{m}_{\mathrm{e}}\right)$-terms generated by the diagrams with the "box" light-by-light-scattering insertions, which form Set III and Set V. In our further considerations we will fix all expressions which are related to the appearance of the diagrams which generate these sets by their numerical values, which are obtained either from exact calculations, or from related estimates. However, we will assume, that after adding the diagrams, which appear in higher orders of perturbation theory and belong to these "non-typical" sets, the method of renormalization group will be valid.

Thus we will assume, that the following contributions to $a_{\mu}$

$$
\begin{aligned}
a_{\mu}^{(I)}(a) & =d_{0}^{(I)} a\left(1+d_{1}^{(I)} a+d_{2}^{(I)} a^{2}+d_{3}^{(I)} a^{3}+\ldots\right) \\
a_{\mu}^{(I I)}(a) & =d_{0}^{(I I)} a^{3}\left(1+d_{1}^{(I I)} a+\ldots\right) \\
a_{\mu}^{(I I I)}(a) & =d_{0}^{(I I I)} a^{4}(1+\ldots)
\end{aligned}
$$

obey the standard RG equations in the on-shell (OS) scheme

$$
\left(m^{2} \frac{\partial}{\partial m^{2}}+\beta(a) \frac{\partial}{\partial a}\right) a_{\mu}^{(I, I I, I I I)}(a)=0
$$

with the QED RG $\beta$-function defined as

$$
m^{2} \frac{\partial a}{\partial m^{2}}=\beta(a)=\beta_{0} a^{2}\left(1+c_{1} a+\sum_{i \geq 1} c_{i} a^{i+2}\right)
$$

where $\beta_{0}=1 / 3, c_{1}=3 / 4, c_{2}=-1.26$ and $c_{3}=-1.713[31$.

Note, that rigorously speaking, this assumption is not proved within the approach, developed in Ref. 44] for the calculation of the asymptotic contributions to $a_{\mu}$ of the subsets of diagrams with internal photon line insertion dressed by electron loops inserted into the muon vertex (this method was used for the analytical evaluation of the specific 10-th order diagrams in Refs. [45, 46]. Some of the results were confirmed later on in Refs. [47, 48, 32. For a recent comparison of the results of analytical calculations of Ref. 46] and the numerical calculations of Ref.[32], see Ref. [49]).

However, in previous studies of the behavior of the QED contributions to $a_{\mu}$ containing light-by-light-scattering terms application of the RG equations gave results which were not inconsistent with the final 10-th order estimate (see e.g. Eq.(14)). In view of this we will continue to use them in our improved considerations, keeping in mind our main aim: to estimate the 5loop contributions to $a_{\mu}^{(I, I I, I I I)}$ using the known numerical values of the lower-order coefficients $\left.\left.d_{i}^{(I)}(0 \leq i \leq 3)\right), d_{i}^{(I I)}(0 \leq i \leq 1)\right), d_{0}^{(I I I)}$ and the coefficients of the QED $\beta$-function in the on-shell scheme.

In our improved studies we will use the following numerical expressions for Eqs.(22)-(24)

$$
a_{\mu}^{(I)}(a)=0.5 a+0.77 a^{2}+3.10 a^{3}+9.11 a^{4}
$$




$$
\begin{aligned}
a_{\mu}^{(I I)}(a) & =20.95 a^{3}+126.27 a^{4} \\
a_{\mu}^{(I I I)}(a) & =-4.43 a^{4}
\end{aligned}
$$

where the coefficients are fixed by careful inspection of the results from Refs. [10, 11] and Refs. 33], 32].

We will review now how the estimates procedure developed in Ref. [22] may be realized using the ideas of the effective charges approach of Ref. [26]. Within this approach one should nullify all higher-order corrections to physical quantities and define for them corresponding effective $\beta$-functions. In the case of $a_{\mu}$ we can write the system of the RG-equations

$$
\begin{aligned}
a_{\mu}^{(I)}\left(a_{E C H}^{(I)}\right) & =d_{0}^{(I)} a_{E C H}^{(I)}(a) \\
\beta_{e f f}\left(a_{E C H}^{(I)}\right) & =\frac{\partial a_{E C H}^{(I)}}{\partial a} \beta(a) \\
a_{\mu}^{(I I)}\left(a_{E C H}^{(I I)}\right) & =d_{0}^{(I I)} a_{E C H}^{(I I) 3}(a) \\
\beta_{e f f}\left(a_{E C H}^{(I I)}\right) & =\frac{\partial a_{E C H}^{(I I)}}{\partial a} \beta(a) \\
a_{\mu}^{(I I I)}\left(a_{E C H}^{(I I I)}\right) & =d_{0}^{(I I I)} a_{E C H}^{(I I I)}(a) \\
\beta_{e f f}\left(a_{E C H}^{(I I I)}\right) & =\frac{\partial a_{E C H}^{(I I I)}}{\partial a} \beta(a) .
\end{aligned}
$$

The $\beta$-functions of the effective charges can be expressed as

$$
\begin{aligned}
& \beta_{e f f}\left(a_{E C H}^{(I)}\right)=-\beta_{0} a_{E C H}^{(I) 2}\left(1+c_{1} a_{E C H}^{(I)}+\sum_{i \geq 2} \tilde{c}_{i}^{(I)} a_{E C H}^{(I) i}\right) \\
& \beta_{e f f}\left(a_{E C H}^{(I I)}\right)=-\beta_{0} a_{E C H}^{(I I) 2} 2\left(1+c_{1} a_{E C H}^{(I I)}+\sum_{i \geq 2} \tilde{c}_{i}^{(I I)} a_{E C H}^{(I I) i}\right) \\
& \beta_{e f f}\left(a_{E C H}^{(I I)}\right)=-\beta_{0} a_{E C H}^{(I I I) 2} 2\left(1+c_{1} a_{E C H}^{(I I I)}+\ldots\right)
\end{aligned}
$$

where $\tilde{c}_{i}^{(I)}$ and $\tilde{c}_{i}^{(I I)}$ coefficients are scheme-invariant and are defined using the general expressions given in Ref. 22.

Let us now, following the considerations of Ref. [24, 22], re-expand $a_{\mu}^{(I, I I, I I I)}\left(a_{E C H}^{(I, I I, I I)}\right)$ in terms of the coupling constant $a$. In this case we will reproduce the known terms of Eqs.(22)(24) and will get extra contributions, namely

$$
\begin{aligned}
a_{\mu}^{(I)}\left(a_{E C H}^{(I)}\right) & =a_{\mu}^{(I)}(a)+\Delta_{N}^{(I)} a^{N+1} \\
a_{\mu}^{(I I)}\left(a_{E C H}^{(I I)}\right) & =a_{\mu}^{(I I)}(a)+\Delta_{N}^{(I I)} a^{N+1} \\
a_{\mu}^{(I I I)}\left(a_{E C H}^{(I I I)}\right) & =a_{\mu}^{(I I I)}(a)+\Delta_{N}^{(I I I)} a^{N+1}
\end{aligned}
$$

which in accordance with the logic of the RG-inspired procedure of estimates of higher-order terms of Ref. 22] will be considered as the estimates of the high order contributions from these 
three sets of diagrams. Their general expressions were derived in Refs. [22, 30] and have the following form

$$
\begin{aligned}
\Delta_{2}^{(I)} & =d_{0}^{(I)} d_{1}^{(I)}\left(c_{1}+d_{1}^{(I)}\right) \\
\Delta_{3}^{(I)} & =d_{0}^{(I)} d_{1}^{(I)}\left(c_{2}-\frac{1}{2} c_{1} d_{1}^{(I)}-2 d_{1}^{(I) 2}+3 d_{2}^{(I)}\right) \\
\Delta_{4}^{(I)} & =d_{0}^{(I)} d_{4}^{(I)}=\frac{d_{0}^{(I)}}{3}\left(3 c_{3} d_{1}^{(I)}+c_{2} d_{2}^{(I)}-4 c_{2} d_{1}^{(I) 2}+2 c_{1} d_{1}^{(I)} d_{2}^{(I)}-c_{1} d_{3}^{(I)}+14 d_{1}^{(I) 4}\right. \\
& \left.-28 d_{1}^{(I) 2} d_{2}^{(I)}+5 d_{2}^{(I) 2}+12 d_{1}^{(I)} d_{3}^{(I)}\right) \\
\Delta_{4}^{(I I)} & =d_{0}^{(I I)} d_{2}^{(I I)}=\frac{2}{3} d_{0}^{(I I)} d_{1}^{(I I) 2}+d_{0}^{(I I)} d_{1}^{(I I)} c_{1} \\
\Delta_{4}^{(I I I)} & =d_{0}^{(I I I)} d_{1}^{(I I I)}
\end{aligned}
$$

The coefficients $d_{i}(0 \leq i \leq 4)$ can be defined as

$$
\begin{aligned}
d_{0}^{(I)} & =B_{1} \\
d_{0}^{(I)} d_{1}^{(I)} & =B_{2}+C_{2} \ln (x) \\
d_{0}^{(I)} d_{2}^{(I)} & =B_{3}+C_{3} \ln (x)+D_{3} \ln ^{2}(x) \\
d_{0}^{(I)} d_{3}^{(I)} & =B_{4}+C_{4} \ln (x)+D_{4} \ln ^{2}(x)+E_{4} \ln ^{3}(x) \\
d_{0}^{(I)} d_{4}^{(I)} & =B_{5}+C_{5} \ln (x)+D_{5} \ln ^{2}(x)+E_{5} \ln ^{3}(x)+F_{5} \ln ^{4}(x) \\
d_{0}^{(I I)} & =\bar{B}_{1} \\
d_{0}^{(I I)} d_{1}^{(I I)} & =\bar{B}_{2}+\bar{C}_{2} \ln (x) \\
d_{0}^{(I I)} d_{2}^{(I I)} & =\bar{B}_{3}+\bar{C}_{3} \ln (x)+\bar{D}_{3} \ln ^{2}(x) . \\
d_{0}^{(I I I)} & =\tilde{B}_{1} \\
d_{0}^{(I I I)} d_{1}^{(I I I)} & =\tilde{B}_{2}+\tilde{C}_{2} \ln (x)
\end{aligned}
$$

where $\ln (\mathrm{x})=\ln \left(\mathrm{m}_{\mu} / \mathrm{m}_{\mathrm{e}}\right) \approx 5$.33. The coefficients $C_{i}, D_{i}, E_{i}, \bar{C}_{i}, \bar{D}_{i}$ and $\tilde{C}_{2}$ are related to the coefficients of the QED $\beta$-function in the OS-scheme as

$$
\begin{gathered}
C_{2}=2 \beta_{0} B_{1} \\
C_{3}=4 \beta_{0} B_{2}+2 \beta_{1} B_{1} \\
D_{3}=4 \beta_{0}^{2} B_{1} \\
C_{4}=6 \beta_{0} B_{3}+4 \beta_{1} B_{2}+2 \beta_{2} B_{1} \\
D_{4}=12 \beta_{0}^{2} B_{2}+10 \beta_{0} \beta_{1} B_{1} \\
E_{4}=8 \beta_{0}^{3} B_{1} \\
C_{5}=8 \beta_{0} B_{4}+6 \beta_{1} B_{3}+4 \beta_{2} B_{2}+2 \beta_{3} B_{1} \\
D_{5}=24 \beta_{0}^{2} B_{3}+28 \beta_{0} \beta_{1} B_{2}+6 \beta_{1}^{2} B_{1}+12 \beta_{0} \beta_{2} B_{1} \\
E_{5}=32 \beta_{0}^{3} B_{2}+\frac{104}{3} \beta_{0}^{2} \beta_{1} B_{1} \\
F_{5}=16 \beta_{0}^{4} B_{1}
\end{gathered}
$$




$$
\begin{aligned}
\bar{C}_{2} & =6 \beta_{0} \bar{B}_{1} \\
\bar{C}_{3} & =8 \beta_{0} \bar{B}_{2}+6 \beta_{1} \bar{B}_{1} \\
\bar{D}_{3} & =24 \beta_{0}^{2} \bar{B}_{1} \\
\tilde{C}_{2} & =8 \beta_{0} \tilde{B}_{1} .
\end{aligned}
$$

It is worth to stress, that Eqs.(44)-(46) reproduce all RG-controllable $\ln (\mathrm{x})$-terms, defined by Eqs.(48)-(52) and in definite cases these terms dominate over the contribution of the constant terms. In view of this, neglecting the unknown constant term $\tilde{B}_{2}$, we can write the RG-inspired estimate for the $\Delta_{4}^{(I I I)}$-coefficient in the following form

$$
\Delta_{4}^{(I I I)} \approx 8 d_{0}^{(I I I)} \beta_{0} \ln \left(\mathrm{m}_{\mu} / \mathrm{m}_{\mathrm{e}}\right)
$$

\subsection{Practical applications}

Let us consider the application of the scheme-invariant procedure to the estimate of the 6-th and 8-th order -contribution to $a_{\mu}$. Using the numbers given in Eq.(27)-Eq.(29)

$$
a_{\mu}^{(I)}(a)=0.5 a+0.77 a^{2}+1.76 a^{3}+9.25 a^{4}
$$

where the $a^{3}$ and $a^{4}$ coefficients were estimated using Eq.(39) and Eq.(40) respectively. Note, that the $a^{3}$ and $a^{4}$ coefficients of this series (namely 1.76 and 9.25) should be compared with the analogous real physical numbers 3.10 and 9.11 (see Eq.(27)).

Being inspired by the satisfactory agreement of the results of applications of this approach (namely by the fact that it gives correct signs and reasonable values of the estimated $\alpha^{3}$ and $\alpha^{4}$ QED coefficients in Eq. (54)) and by its interesting outcomes in QCD (see Ref. 30]), we will apply it to get the improved estimate the 10-th order QED contribution to $a_{\mu}$ of the first three terms in Eq.(21) using Eq.(41), Eq.(42) and Eq.(43) respectively. The results of these new estimates are:

$$
\begin{aligned}
\Delta_{(10)}^{(I)} & =\Delta_{4}^{(I)} \approx 32 \\
\Delta_{(10)}^{(I I)} & =\Delta_{4}^{(I I)} \approx 602 \\
\Delta_{(10)}^{(I I I)} & =\Delta_{4}^{(I I I)} \approx-63 .
\end{aligned}
$$

Summing them with the results of recent direct calculations from Ref. 32] of the sets of the diagrams, shown in Fig.3 and Fig.4, namely

$$
\begin{aligned}
\Delta_{(10)}^{(I V)} & =97.123(62) \\
\Delta_{(10)}^{(V)} & =-25.506(20)
\end{aligned}
$$

we obtain the following estimate of the RG-inspired approach

$$
\Delta_{(10)}(\mathrm{est}) \approx 643
$$


Note that this estimated number turned out to be in good agreement with the final result of Ref. 32

$$
\Delta_{(10)}(\text { calc })=663(20)
$$

This fact allows us to make the statement that Kinoshita and Nio really considered 2958 graphs, which play dominant role in forming 10-th order corrections to the whole $\alpha^{5}$ coefficient, and that still uncalculated 6122 graphs, not taken into account in the process of obtaining the estimate of Eqs.61), may be not important.

\section{Comparison with other related results}

Let us first mention the sours of improvements of the order $a^{5}$-coefficients to $a_{\mu}$, estimated in Ref. [22. Note, that the new estimate of Eq. (55) is slightly smaller than the similar old estimate of Ref. $22, \Delta_{4}^{(I)} \approx 50$. This effect of reduction is explained by the fact that in the process of analysis the improved four-loop results for $a_{e}$, obtained in Ref. 35], were taken into account. Moreover, definite numerical bugs, which crept in the process of getting numerical number of Eq.(13), were eliminated.

It is also quite understandable, why the coefficient in Eq.(14) differs from the similar one, obtained in this work. Indeed, this analog was obtained using the improved calculations of Ref. [34. Moreover, the presented in Eq.(14) result of Ref. [22] should be compared with the sums of Eq.(56) and Eq.(57) and this gives reasonable agreement of numbers (520 vs 539).

And finally, the used in this work value of Eq.(58), which comes from the explicit calculations of Ref. 32, is smaller than its estimate, given in Ref. [18] (see Eq.(10)). This difference (88) is explaining us the reason of the finding analogous decreasing effect in the coefficient $\Delta_{(10)}(e s t)$ (compare Eq.(15) with Eq.(60)).

It is also interesting to compare other discussed in this work approximate results with the explicit results, which exist in Ref. [32]. First, using the renormalization-group formalism it is possible to derive the following expression for the sum of diagrams, which contain the diagram of Fig.2 22]:

$$
a_{\mu}(\text { Fig. } 2)=\left(\overline{\mathrm{B}}_{2}(\text { Fig. } 2)+6 \overline{\mathrm{B}}_{1} \beta_{1} \ln \left(\mathrm{m}_{\mu} / \mathrm{m}_{\mathrm{e}}\right)\right)\left(\frac{\alpha}{\pi}\right)^{5}
$$

where $\bar{B}_{1}=d_{0}^{(I I)}=20.9$ is the approximate expression of the three-loop light-by-light scattering graph with electron loop and muon vertex and $\beta_{1}=1 / 4$ is the two-loop coefficient of the QED $\beta$-function, which is defined from the two-loop photon-vacuum polarization insertion. Thus the total value of the $\ln$-contribution to Eq.(62) is $6 \bar{B}_{1} \beta_{1} \ln \left(\mathrm{m}_{\mu} / \mathrm{m}_{\mathrm{e}}\right)=167$ while the estimate of the whole coefficient in Eq.(62) given in Ref. 18 is $176 \pm 35$ almost coincide with this number. Moreover, the explicit calculations of Ref. 32 which gives for this contribution the number 181.1285(51) is supporting both the estimates of Ref. 32] and the renormalizationgroup technique, used throughout this work and leaves the room for positive contribution of the constant term $\bar{B}_{2}$ (Fig.2). It is also interesting that the renormalization-group inspired method is giving rather precise estimate for the five-loop diagrams, which contribute in Eq.(57), namely 
the dressed by one electron loop contribution internal photon lines, which connect the electron "box" insertion and two muon lines of the external vertex. The RG-inspired formalism gives for this contribution the estimate $\Delta_{(10)}^{(I I I)} \approx-63$ while the explicitly calculated in Ref. [32] results is $\Delta_{(10)}^{(I I I)}=-57.0633(109)$. This is one more example, when explicate calculations are supporting the RG-inspired approach of Ref. [22] used in our work.

For the completeness of the discussions is also worth to mention that some attempts to improve the precision of the truncated perturbative expansion were made in Ref. [50] by means of the Pade resummation technique. However, it is difficult to compare their estimates with the results discussed in this paper. Indeed, the analysis of Ref. [50] does not allow to separate the renormalization-group controllable contributions from the ones, which are coming from the diagrams with the light-by-light-type structure.

To conclude, we see, that the renormalization-group inspired scheme-invariant approach is working quite satisfactory for the estimates of 10-th order corrections to the muon anomalous magnetic moment. The estimates obtained are supporting the applications of the results of recent calculations of Ref. 32] in the phenomenological analysis. The current difference between phenomenological and experimental results for $a_{\mu}$ (see e.g. Ref.[51]), which is more straightforwardly related to the values of the effects of strong interactions, can not be described by including tenth order QED effects in the theoretical predictions. Indeed, they are smaller than the experimental uncertainties. Thus the present value of the order $a^{5}$ QED contribution to $a_{\mu}$ is over 0.01 times smaller than the previous order $a^{4}$-one, which in tern is over 0.013 times smaller than the order $a^{3}$-contribution. The numerical values of these terms, namely $0.4 \times 10^{-10}$ and $38.1 \times 10^{-10}[52$ may serve the aim for fixing the errors of possible future $\left(g_{\mu}-2\right)$ measurements, since most recent one has statistical and systematical uncertainties of over $( \pm 5.4 \pm 3.3) \times 10^{-10}\left[51\right.$, which are higher that the order $\alpha^{5}$ QED correction.

\section{Acknowledgments}

I would like to thank T. Kinoshita for renewing my interest to the considerations of the 10-th order QED corrections to $a_{\mu}$, for inviting me to present the results of these studies at the Cornell University and for useful discussions. I am grateful to S.L. Adler for his invitation to visit Institute for Advanced Study in Princeton, where this work was started. I want to wish the success to V.V Starshenko in his new work at IPG Photonic Corporation. The collaboration with him at the previous stage of the analogous studies was really pleasant. It is really worth to acknowledge the encouraging atmosphere of Theoretical Division of INR. This work is supported by RFBR Grant N 05-01-00992. 


\section{References}

[1] R. P. Feynman, "The Present Status Of Quantum Electrodynamics," Talk at the 1961 Solvey Conference; See "Selected Papers of Richard Feynman", Ed. L.M. Braun, World Scientific, 2000, p.141.

[2] J. S. Schwinger, Phys. Rev. 73, 416 (1948).

[3] C. S. Sommerfield, Phys. Rev. 107, 328 (1957).

[4] A. Petermann, Helv. Phys. Acta XXX, 407 (1957).

[5] M. V. Terentiev, Zh. Eksp. Teor. Fiz. 43619 (1962)[ Sov. Phys. JETP 16, 444 (1963)].

[6] S. D. Drell and H. R. Pagels, Phys. Rev. 140, B397 (1965).

[7] R. G. Parsons, Phys. Rev. 168, 1562 (1968).

[8] J. A. Mignaco and E. Remiddi, Nuov. Chim. A LX , 519 (1969).

[9] J. Aldins, T. Kinoshita, S. J. Brodsky and A. J. Dufner, Phys. Rev. D 1, 2378 (1970).

[10] S. Laporta and E. Remiddi, Phys. Lett. B 265, 182 (1991).

[11] S. Laporta and E. Remiddi, Phys. Lett. B 379, 283 (1996) arXiv:hep-ph/9602417.

[12] M. Veltman, in Proc. of III Int. Workshop on Software Engineering, Artificial Intelligence and Expert Systems for HE and Nuclear Physics, Obberammergau, October 1993, Germany, World Scientific, p.451.

[13] L. N. Lipatov, private communication.

[14] T. Kinoshita, B. Nizic and Y. Okamoto, Phys. Rev. D 41593 (1990).

[15] T. Kinoshita, Phys. Rev. Lett. 61, 2898 (1988).

[16] S. Laporta and E. Remiddi, Phys. Lett. B 301, 440 (1993).

[17] J. H. Kuhn, A. I. Onishchenko, A. A. Pivovarov and O. L. Veretin, Phys. Rev. D 68, 033018 (2003) arXiv:hep-ph/0301151.

[18] S. G. Karshenboim, Yad. Fiz. 56, 252 (1993) [ Phys. Atom. Nucl. 56, 857 (1993)].

[19] A. S. Elkhovskii, Yad. Fiz. 49, 1059 (1989) [ Sov. J. Nucl. Phys. 49, 656 (1989)]

[20] A. I. Milstein and A. S .Yelkhovsky, Phys. Lett. B 233, 11 (1989).

[21] A. Czarnecki and W. J. Marciano, Phys. Rev. D 64, 013014 (2001) arXiv:hep-ph/0102122.

[22] A. L. Kataev and V. V. Starshenko, Phys. Rev. D 52, 402 (1995) arXiv:hep-ph/9412305. 
[23] N. N. Bogolyubov and D. V. Shirkov, "Introduction To The Theory Of Quantized Fields," Nauka, Moscow, 1957; translated by Willey Interscience as Interscience monographs and texts in Physics and astronomy 3 (1959) 1.

[24] P. M. Stevenson, Phys. Rev. D 23, 2916 (1981).

[25] G. Grunberg, Phys. Lett. B 95, 70 (1980).

[26] G. Grunberg, Phys. Rev. D 29, 2315 (1984).

[27] N. V. Krasnikov, Nucl. Phys. B 192 (1981) 497

[28] A. L. Kataev, N. V. Krasnikov and A. A. Pivovarov, Phys. Lett. B 107, 115 (1981).

[29] A. L. Kataev, N. V. Krasnikov and A. A. Pivovarov, Nucl. Phys. B 198, 508 (1982) [Erratum-ibid. B 490, 505 (1997)] arXiv:hep-ph/9612326.

[30] A. L. Kataev and V. V. Starshenko, Mod. Phys. Lett. A 10, 235 (1995) arXiv:hep-ph/9502348.

[31] D. J. Broadhurst, A. L. Kataev and O. V. Tarasov, Phys. Lett. B 298, 445 (1993) arXiv:hep-ph/9210255.

[32] T. Kinoshita and M. Nio, Phys. Rev. D 73, 053007 (2006); hep-ph/0512330.

[33] M. Passera, J. Phys. G 31, R75 (2005) arXiv:hep-ph/0411168.

[34] T. Kinoshita and M. Nio, Phys. Rev. D 70, 113001 (2004) arXiv:hep-ph/0402206.

[35] T. Kinoshita and M. Nio, Phys. Rev. D 73, 013003 (2006) arXiv:hep-ph/0507249.

[36] G. Gabrielse, D. Hanneke, T. Kinoshita, M. Nio and B. Odom1, Phys. Rev. Lett. 97, 030802 (2006).

[37] T. Kinoshita, Nucl. Phys. Proc. Suppl. 144, 206 (2005).

[38] Some numerical results announced in Ref. 37] were improved later on in the final publication of Ref. 32 .

[39] A. L. Kataev, Nucl. Phys. Proc. Suppl. 155, 369 (2006), hep-ph/0507174.

[40] M. Passera, hep-ph/0606174.

[41] S. J. Brodsky, G. P. Lepage and P. B. Mackenzie, Phys. Rev. D 28, 228 (1983).

[42] B. E. Lautrup and M. A. Samuel, Phys. Lett. B 72, 114 (1977).

[43] E. A. Kuraev, Z. K. Silagadze, A. A. Cheshel and A. Schiller, Sov. J. Nucl. Phys. 50, 264 (1989) [Yad. Fiz. 50, 422 (1989)].

[44] B. Lautrup and E. De Rafael, Nucl. Phys. B 70, 317 (1974). 
[45] A. L. Kataev, Pisma Zh. Eksp. Teor. Fiz. 54, 600 (1991) [ JETP Lett. 54, 602 (1991)]

[46] A. L. Kataev, Phys. Lett. B 284, 401 (1992).

[47] D. J. Broadhurst, Z. Phys. C 58, 339 (1993).

[48] S. Laporta, Phys. Lett. B 328, 522 (1994) arXiv:hep-ph/9404204.

[49] A. L. Kataev, Proceedins of the 12th Lomonosov Conference on Elementary Particle Physics, Moscow State University, Moscow, 25-31 August 2005 (to be published); hep-ph/0602098.

[50] J. R. Ellis, M. Karliner, M. A. Samuel and E. Steinfelds, hep-ph/9409376.

[51] G. W. Bennett et al. [Muon G-2 Collaboration], Phys. Rev. D 73, 072003 (2006) arXiv:hep-ex/0602035.

[52] S.I. Eidelman, talk at Parallel Session 6 of ICHEP-06 Conference, Moscow, July-August, 2006; (to be published). 